the closed sets. Notice that according to (iv), most spaces of importance in mathematics are $c$-spaces.

A category of antispaces $T$ and onto continuous mappings $f$ corresponds to the category of spaces $T^{*}$ and onto mappings $f^{*}\left(f^{*}\right.$ defined by the requirement, that the inverse of any compact image set is compact). This sets up an isomorphism as mentioned in the title.

UNIVERSITY OF AMSTERDAM AND

UNIVERSITY OF FLORIDA

\title{
SOME EXPLICIT POLYNOMIAL APPROXIMATIONS IN THE COMPLEX DOMAIN
}

BY T. J. RIVLIN

Communicated by R. C. Buck, January 20, 1967

1. Let $f(z)$ be continuous on a compact set, $B$, in the complex plane. Then if $B$ contains more than $n$ points $f(z)$ has a unique best uniform approximation out of the polynomials of degree $n$. That is, if $P_{n}$ is the set of polynomials of degree at most $n$, there exists $p_{n}^{*} \in P_{n}$ satisfying

$$
\left\|f-p_{n}^{*}\right\|<\|f-p\|
$$

for all $p \in P_{n}, p \neq p_{n}^{*}$, the norm being the uniform norm. It is an instance of a result due to Kolmogorov (see Meinardus [2; p. 15], for example) that $p_{n}^{*} \in P_{n}$ satisfies (1) if, and only if,

$$
\min _{z \in E} \operatorname{Re}\left[f(z)-p_{n}^{*}(z)\right] p(\bar{z}) \leqq 0, \quad p \in P_{n},
$$

where

$$
E=\left\{z /\left|f(z)-p_{n}^{*}(z)\right|=\left\|f-p_{n}^{*}\right\|\right\}
$$

Let us put

$$
\rho_{n}(f ; B)=\left\|f-p_{n}^{*}\right\| .
$$

In [1] $\mathrm{Al}^{\prime}$ per showed that

$$
\rho_{n}\left(z^{s} /\left(z^{p}-a^{p}\right) ; K_{R}\right)=R^{p k+p+s} /\left(|a|^{2 p}-R^{2 p}\right)|a|^{p k}
$$


where $K_{R}$ is the disc $|z| \leqq R,|a|>R, p$ is a positive integer, $s=0$, $1, \cdots, p-1$ and $n=p k+s, p k+s+1, \cdots, p k+s+p-1$ for every nonnegative integer $k$.

It is the purpose of this note to exhibit the polynomials $p_{n}^{*}$ for which (5) is attained. To our knowledge this is the first example of the explicit construction of relatively nontrivial best uniform polynomial approximations in the complex domain.

2. The essential part of our result is contained in the

TheOREM. If $0<A<1$, the best uniform approximation by polynomials of degree at most $n$ on $K:|z| \leqq 1$ to the function

$$
f(z)=1 /(1-A z)
$$

is

$$
q_{n}(z)=1+A z+A^{2} z^{2}+\cdots+A^{n-1} z^{n-1}+\left(A^{n} /\left(1-A^{2}\right)\right) z^{n} .
$$

Proof.

$$
f(z)-q_{n}(z)=\left(A^{n+1} /\left(1-A^{2}\right)\right) z^{n}(z-A) /(1-A z) .
$$

We can now either refer the reader to $\mathrm{Al}^{\prime}$ per [1] or conclude as follows. The set $E$ needed in (2) and defined in (3) is, for $f-q_{n}$, clearly $C:|z|=1$. Given $p \in P_{n}$, on $|z|=1$

$$
\begin{aligned}
\operatorname{Re}\left[f(z)-q_{n}(z)\right] p(\bar{z}) & =\left(A^{n+1} /\left(1-A^{2}\right)\right) \operatorname{Re}\left[\bar{z}^{n}(\bar{z}-A) /(1-A \bar{z})\right] p(z), \\
& =\left(A^{n+1} /\left(1-A^{2}\right)\right) \operatorname{Re}\left[z^{-n}(1-A z) /(z-A] p(z),\right.
\end{aligned}
$$

and hence

$$
\min _{z \in C} \operatorname{Re}\left[f(z)-q_{n}(z)\right] p(\bar{z})=\frac{A^{n+1}}{1-A^{2}} \min _{z \in C} \operatorname{Re}\left[\left(\frac{z-A}{1-A z}\right) z^{n} p\left(\frac{1}{z}\right)\right] .
$$

But $\operatorname{Re}\left[((z-A) /(1-A z)) z^{n} p(1 / z)\right]$ is harmonic in $|z| \leqq 1$ and has a zero at $A$, therefore its minimum on $|z|=1$ cannot be positive. In view of condition (2) the Theorem is thus proved.

Corollary 1.

$$
\rho_{n}(1 /(1-A z) ; K)=A^{n+1} /\left(1-A^{2}\right),
$$

which agrees with (5).

CoRollary 2. If $0<A<1$, the best uniform approximation by polynomials of degree at most $n$, where $k p+s \leqq n<(k+1) p+s$, on $K:|z| \leqq 1$ to

$$
f(z)=z^{s} /\left(1-A^{p} z^{p}\right),
$$


where $p$ is a positive integer, $s=0,1, \cdots, p-1$, and $k$ is a given nonnegative integer, is the function

$$
\begin{aligned}
q(z)=z^{s}\left(1+A^{p} z^{p}+A^{2 p} z^{2 p}+\cdots+A^{(k-1) p} z^{(k-1) p}\right. & \\
& \left.+\left(A^{k p} /\left(1-A^{2 p}\right)\right) z^{k p}\right) .
\end{aligned}
$$

(Note that it suffices to prove this for $n=k p+s$. The full result then follows from the observation that since $\left(1-A^{p} z^{p}\right)^{-1}$ is a function of $z^{p}$, only powers of $z^{p}$ can appear in its polynomials of best approximation.) It is easy to extend these results to $|z| \leqq R$, and thereby recover (5).

\section{REFERENCES}

1. S. Ja. Al'per, Asymptotic values of best approximation of analytic functions in a complex domain, Uspehi Mat. Nauk 14 No. 1(85) (1959), 131-134.

2. G. Meinardus, Approximation von Funktionen und ihre numerische Behandlung, Springer, New York, 1964.

ibM Watson Research Center, Yorktown Heights, New York 